anthropology \& materialism

\section{Anthropology \& Materialism}

A Journal of Social Research

$4 \mid 2019$

Art and Technique: A Framework of Unaccomplished Promises

\title{
L'avenir du travail selon Google
}

The Future of Work according to Google

El futuro del trabajo según Google

\section{Sébastien Broca}

Édition électronique
URL : http://journals.openedition.org/am/1255

DOI : $10.4000 /$ am. 1255

ISSN : 2364-0480

\section{Éditeur :}

CETCOPRA, CRASSH - Center for Research in the Arts Social Sciences and Humanities, Fakultät Gestaltung - Universität der Künste Berlin

Référence électronique

Sébastien Broca, «L'avenir du travail selon Google », Anthropology \& Materialism [En ligne], 4 | 2019, mis en ligne le 11 novembre 2019, consulté le 23 novembre 2019. URL : http://journals.openedition.org/ am/1255; DOl : 10.4000/am.1255

Ce document a été généré automatiquement le 23 novembre 2019.

Tous droits réservés 


\section{L'avenir du travail selon Google}

The Future of Work according to Google

El futuro del trabajo según Google

\section{Sébastien Broca}

\section{NOTE DE L'AUTEUR}

Cet article est une version légèrement réécrite d'une présentation donnée le 8 novembre 2016 à l'Université Nationale de Rosario (Argentine) dans le cadre de la rencontre Fotogramas del pensiamento.

This article is a slightly rewritten version of a presentation I made in November 2016 at the Universidad Nacional de Rosario in Argentina during the conference Fotogramas del pensiamento.

Este artículo corresponde a una versión levemente modificada de una presentación realizada el 8 de noviembre de 2016 en la Universidad Nacional de Rosario, en el marco del coloquio Fotogramas del pensamiento. 


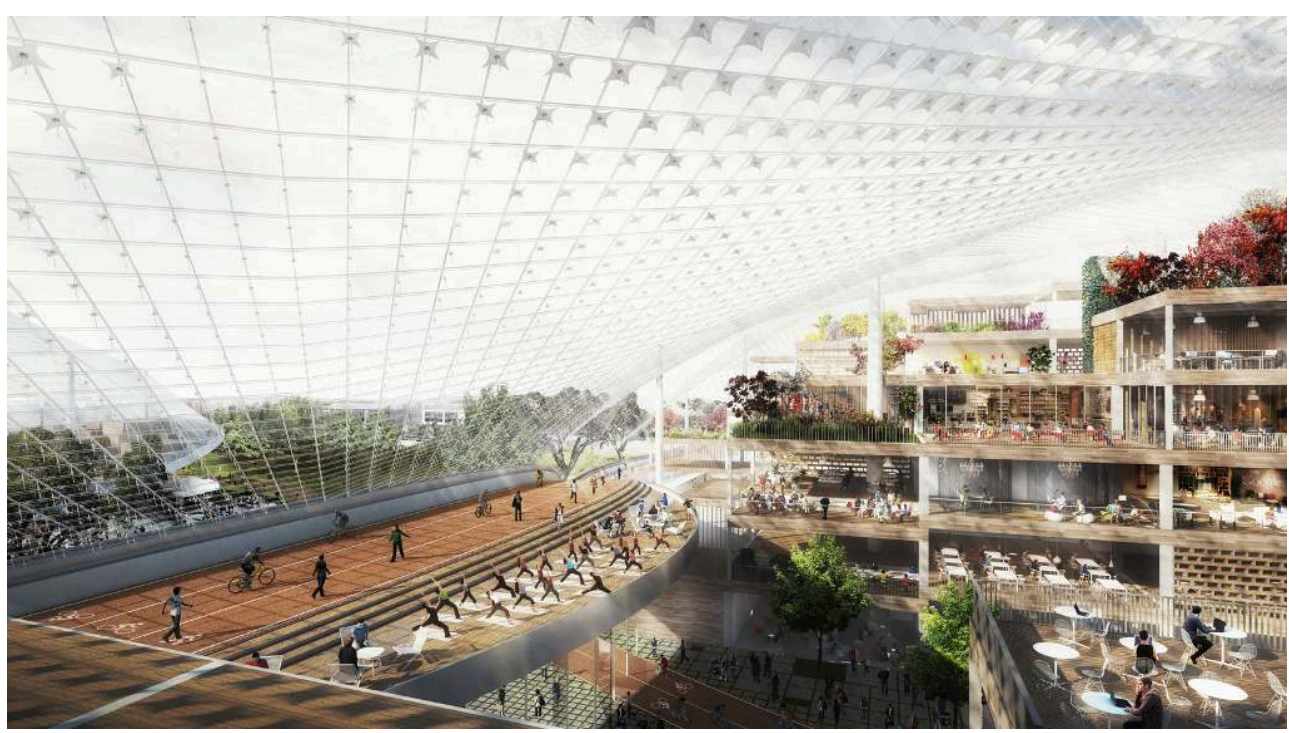

Image Reference : Bjarke Ingels Group (BIG) \& Heatherwick Studio, " Google North Bayshore_8 », 2015

http://www.big.dk/\#projects-gccp

\section{Introduction}

1 L'image numérique ci-dessus a été produite en 2015 par un cabinet d'architectes danois (Bjarke Ingels Group) et un studio de design anglais (Thomas Heatherwick), choisis par Google pour concevoir ensemble les plans du futur campus de l'entreprise à Mountain View en Californie. Elle fait partie d'une série présentant le complexe architectural et les diverses activités destinées à y prendre place. Le campus a été pensé pour héberger les équipes dirigeantes de Google et un grand nombre de salariés (environ 10000 personnes), chargés notamment de développer les produits et les services les plus ambitieux de la firme : drones fonctionnant à l'énergie solaire, voitures autonomes, etc.

2 Le projet de Google n'est pas unique en son genre. Plusieurs géants de la Silicon Valley ont travaillé ces dernières années à des bâtiments similaires, avec l'appui de quelques stars de l'architecture : Facebook a enrôlé Frank Gehry pour redessiner son campus, Apple a fait appel à Norman Foster pour construire son nouveau quartier général en forme de gigantesque vaisseau spatial. Le projet architectural et urbanistique de Google, tel qu'il a été présenté en 2015, présente toutefois certains aspects remarquables. Il ne s'agit pas simplement de construire un bâtiment, mais bien d'organiser une véritable ville miniature s'étendant sur $316000 \mathrm{~m}^{2}$. Cette ville s'organise autour de quatre grandes canopées de verre, rappelant quelque peu les fameux dômes géodésiques de Buckminster Fuller. Chacune abrite de grands espaces ouverts propices à de multiples activités : travail, détente, sport, sociabilité, shopping... Selon leurs concepteurs, ces canopées permettent de faire entrer l'air et la lumière de l'extérieur, tout en protégeant des intempéries et en permettant de contrôler la température intérieure. Elles sont installées au sein de gigantesques espaces verts, présentés comme conformes à la nature californienne originelle (le projet parle de " native ecosystems»). On trouve aussi sur le campus des espaces additionnelles de 
bureaux et de réunions, pouvant être déplacés sur toute l'étendue du Googleplex en fonction des besoins. Enfin, 5000 nouveaux logements sont situés à proximité immédiate, afin d'accueillir les salariés de Google et leurs familles.

3 Ce projet ambitieux, tel que je viens de le présenter à gros traits, ne verra jamais le jour sous cette forme exacte. En mai 2015, le conseil municipal de Mountain View a refusé de céder à Google l'ensemble des terrains nécessaire pour construire cette petite ville dédiée à l'innovation (Oremus 2015). L'entreprise et ses architectes ont donc dû restreindre quelque peu leurs ambitions : le complexe actuellement en cours de construction est de taille bien plus modeste. Le projet initial représente quoi qu'il en soit un formidable aperçu de la manière dont la firme de Mountain View se représente l'avenir du travail : son contenu, son organisation et son inscription dans le territoire. Or Google n'est évidemment pas une entreprise comme les autres. Il s'agit, désormais sous le nom d'Alphabet, d'une des plus grandes capitalisations boursières mondiales. $C^{\prime}$ 'est aussi une entreprise dont les services modèlent notre quotidien et dont l'attractivité est très forte chez les jeunes diplômés (Gless 2017). L'environnement de travail de Google suscite du désir, voire une forme de fascination. Il y a donc quelque raison de s'intéresser à la manière dont cette entreprise imagine le futur du travail et on peut penser que cette vision nourrit les représentations sociales, particulièrement au sein des fractions les plus favorisées de la main d'œuvre.

De façon générale, la Silicon Valley est ainsi un territoire qu'il faut aussi aborder comme un «territoire mental» (Sadin 2016). En d'autres termes, la réussite économique d'une entreprise comme Google ne peut être séparée de l'idéologie sur laquelle elle s'appuie (Barbrook et Cameron 1996 ; Morozov 2013). Or cette idéologie est traversée d'éclats d'utopie, c'est-à-dire de projections figurant un monde plus désirable que le nôtre. Les images du futur campus en sont un bon exemple. Je voudrais ici les analyser de manière essentiellement sociologique, en faisant l'hypothèse que le projet conçu par Google et ses architectes dit quelque chose de notre rapport actuel aux transformations du travail liées à l'économie numérique. Ces images peuvent en ce sens être vues comme des fragments d'utopie qui nous parlent d'abord de notre présent. Fredric Jameson rappelle que l'utopie est ce qui révèle " les limites de notre propre imagination du futur, les lignes que nous ne semblons pas capables de franchir en imaginant des changements dans notre vie et notre monde " (Jameson 2007). Il est pour cette raison instructif de s'attarder sur le projet de campus de Google, quand bien même celui-ci ne verra jamais le jour sous cette forme exacte. Si comme l'écrit encore Jameson, « le désir humain est constitutif du projet collectif et de la construction historique des formations sociales» (ibid.), les désirs qui transparaissent dans cette image nous disent quelque chose de l'évolution du travail et des représentations que nous y associons.

\section{Une société miniature}

5 Lorsqu'on considère le projet architectural de Google, il est frappant de constater que l'entreprise ne se représente plus comme une grande organisation hiérarchique (Coase 1937) mais comme une sorte de société miniature. Cet aspect est déjà patent au sein de l'actuel Googleplex, où les employés de Google disposent depuis plusieurs années d'un très grand nombre de services touchant aussi bien à la vie domestique (machines à laver, pressing) qu'à la santé (médecins, dentistes) ou aux loisirs (multiples terrains de 
sport, jeux vidéos, etc.) (Strickland 2008). Le projet présenté par Ingels et Heatherwick pousse néanmoins cette volonté de recréer in vitro tous les aspects de la vie sociale et individuelle à un point paroxystique.

Cette ambition a de nombreux antécédents historiques (et en ce sens, le projet de Google est sans doute moins original que la rhétorique de la disruption, caractéristique de la Silicon Valley, tend à nous le faire croire). On peut y voir un lointain écho de l'utopie phalanstérienne de Fourier, voire de la Cité radieuse de Le Corbusier ou encore de l'édifice de Dessau, conçu par le fondateur du Bauhaus, Walter Gropius, comme un bâtiment où il serait possible aussi bien de travailler que de s'amuser, de manger et de dormir (Berdet 2013, 189). Le projet de Google rappelle aussi, peut-être plus nettement, certaines villes-entreprises bâties au XIX ${ }^{\mathrm{e}}$ siècle par de riches industriels : Hershey en Pennsylvanie, Fordlandia au Brésil (dont on dit qu'elle a inspiré Disneyland), ou encore Pullman dans l'Illinois qui fut fondée par le créateur des wagons-lits du même nom.

Pullman, dans l'Illinois, fut construite sur une petite colline de quatre mille acres de long à côté du lac Calumet juste au sud de Chicago [...]. La ville fut fondée en 1880 et terminée pour l'essentiel, selon un plan unique et unifié, en deux ans. Pullman (le propriétaire) n'y mit pas simplement des usines et des dortoirs [...]. Il construisit des maisons privées, des maisons alignées et des immeubles d'habitation pour environ sept ou huit cents personnes, des boutiques et des bureaux (autour d'une arcade complexe), une bibliothèque, un théâtre et même une église ; en bref une petite ville modèle, une communauté planifiée. Et tout ce qu'il y avait dedans lui appartenait (Walzer 2013, 409-410).

7 La propriété de Pullman sur toutes les infrastructures ne tarda pas à se transformer pour le magnat en la conviction selon laquelle le pouvoir politique était aussi sa chose. Il gérait donc la ville comme un autocrate, contrôlant strictement l'apparence des habitants, leur consommation d'alcool et la rénovation de leurs maisons. En contrepartie, les logements fournis par Pullman étaient confortables et ses travailleurs jouissaient globalement de conditions de vie plus agréables que celles de la majorité des américains de l'époque. L'expérience s'acheva malgré tout en 1898, lorsque la Cour suprême de l'Illinois jugea que la possession d'une ville entière par un industriel était incompatible avec l'esprit des institutions américaines (Ibid., 412-413).

8 Le précédent historique de Pullman est intéressant à plusieurs titres. Cette tentative d'organiser, à travers l'architecture d'une petite ville construite de toute pièce, toutes les sphères de l'existence individuelle et collective évoque assez nettement le projet de Google. Aujourd'hui, certains craignent du reste que les GAFAM déploient un nouveau « paternalisme industriel 2.0» (Maurel 2018), qui s'appliquerait à leurs employés mais progressivement aussi à l'ensemble de leurs utilisateurs, à mesure que reculerait l'État social. Les réticences de la municipalité de Mountain View face au projet initial de Google ne sont pas non plus sans lien avec les raisons qui avaient jadis poussé la Cour suprême de l'Illinois à mettre fin à l'expérience de Pullman. En effet, c'est bien la crainte du poids politique de Google et la peur de devenir une "ville-entreprise », qui ont poussé la municipalité californienne à refuser le gigantesque projet architectural qui lui était soumis (Oremus 2015). 


\section{Une bulle transparente}

9 Il est possible d'inscrire le projet de Google dans une généalogie quelque peu différente, en le rattachant non plus aux exemples de villes-entreprises mais à la tradition des grands campus universitaires américains. Ceux-ci symbolisent l'excellence et la richesse de certaines universités, autant que la singularité du mode de vie étudiant en Amérique de Nord. Ils constituent aussi des sociétés miniatures, isolées de l'extérieur, où tous les besoins individuels peuvent être pris en charge. Ce sont " des mondes à part, des endroits qui sont pensés pour l'étudiant dans sa globalité afin de former l'étudiant dans sa globalité » (Naddaff-Hafrey 2016). On y étudie, on y mange, on y dort, on y fait la fête, on y fait du sport et on s'y engage dans diverses activités culturelles ou politiques, sans jamais avoir besoin de sortir (ou presque). Cette coupure vis-à-vis du monde extérieur est l'une des caractéristiques essentielles des environnements de travail construits par les multinationales de la Silicon Valley. C'est également l'une des choses qui leur est le plus fréquemment reprochée, comme en témoignent les protestations massives contre les « Google bus » à San Francisco, ces cars affrétés par les géants de la tech pour leurs employés, afin de les conduire directement à leur travail sans subir les avanies des transports en commun et les turpitudes du reste de la population (Smyrnaios 2017, 6).

10 Dans le projet présenté en 2015, Google et ses architectes ont toutefois essayé de s'épargner ce type de reproche. Les dirigeants de l'entreprise ont promis que les futurs espaces verts seraient accessibles à tous et $\mathrm{T}$. Heatherwick a assuré que « faire sortir l'organisation du monde n'[était] clairement pas le but " (cité par Stone 2015). La transparence des gigantesques canopées de verre peut apparaître comme la traduction architecturale de cet engagement. Les motifs de la transparence et de l'ouverture caractérisent du reste l'ensemble du campus, dont les différents espaces ne sont jamais séparés de manière étanche. Ce parti-pris architectural a, me semble-t-il, une signification ambivalente. Il évoque certes l'ouverture vers l'extérieur et la volonté de ne pas construire une "forteresse " réservée aux employés de Google. Il suggère également un monde du contrôle permanent, où le public et le privé tendent à se confondre dans la mesure où les actions individuelles sont perpétuellement exécutées sous le regard des autres.

11 Cette deuxième dimension n'est certainement pas étrangère à l'idéologie de Google, dont le modèle économique lui impose de recueillir un maximum de données sur ses utilisateurs et dont l'un des ingénieurs stars (Vinton Cerf) déclarait en 2013 que « la vie privée pourrait bien être une anomalie » (cité par Martin 2013). C'est donc bien la société transparente rêvée par Google qui est construite en modèle réduit dans le projet architectural de B. Ingels et $\mathrm{T}$. Heatherwock. La caractéristique principale de cette société est l'estompement des frontières entre les différentes sphères de l'existence individuelle : travail, loisirs, vie privée, etc.

\section{Un travail biopolitique}

12 Ce brouillage des frontières a un rapport très direct avec les évolutions récentes du travail et de ses représentations. Le travail est de moins en moins considéré comme un pan de l'existence nettement séparé des autres, et ce pour plusieurs raisons convergentes. Tout d'abord, plusieurs activités qui n'étaient auparavant pas vues sous 
l'angle du travail sont désormais fréquemment abordées comme telles. Il en va ainsi du "travail domestique», tel qu'il a été conceptualisé par la critique féministe matérialiste à partir des années 1960, mais aussi du « travail bénévole » (Simonet 2010) ou encore du " travail du consommateur " (Dujarier 2014). Les analystes de l'économie d'Internet ont d'une certaine manière exacerbé cette tendance à étendre la notion de travail et à en effacer la spécificité. Ils ont ainsi créé plusieurs néologismes (prosumption, playbor, weisure), qui viennent brouiller des distinctions auparavant couramment admises : production et consommation, jeu et travail, travail et loisirs.

Ces évolutions récentes ne sont pas uniquement discursives. Le contenu du travail et ses conditions d'exercice ont changé, du moins dans certains secteurs de l'économie où l'unité de temps et de lieu qui caractérisait la majorité des activités productives propres au capitalisme industriel semble s'être dissoute. On ne travaille plus de $9 \mathrm{~h}$ à $17 \mathrm{~h}$ dans un espace dédié à cette activité : le bureau ou l'usine. On emporte du travail avec soi le soir, on travaille depuis son domicile, on répond à ses e-mails professionnels sur son lieu de vacances (et à ses e-mails personnels sur son lieu de travail), on alterne périodes d'activités frénétiques où la journée de travail dure $12 \mathrm{~h}$ et périodes de " creux » où on ne travaille qu'une poignée d'heures par jour... Certes, il est entendu que ces évolutions ne touchent pas uniformément l'ensemble des travailleurs. Elles sont particulièrement caractéristiques des industries culturelles et créatives (Cingolani 2014), ainsi que des formes d'activités associées à l'informatique et au numérique (Himanen 2001), où le cadre rigide du salariat fordiste se révèle parfois peu adapté.

Pour un certain nombre de travailleurs de ces secteurs, les représentations et les attentes liées au travail ont également changé. Le travail est moins abordé comme un moyen de gagner sa vie et davantage comme un vecteur de réalisation de soi. Se trouvent ainsi importées dans la sphère professionnelle des attentes qui relevaient autrefois essentiellement de la sphère familiale et des loisirs, évolution qui est loin d'être uniquement positive. Cela peut être source de frustration, voire d'aliénation, lorsque les conditions de travail empêchent d'y trouver l'accomplissement qu'on y recherchait. L'horizon d'accomplissement personnel dans le travail représente en outre un formidable outil de mobilisation et de motivation des individus. Il fonctionne comme une incitation à travailler davantage, dès lors que c'est l'ensemble de la subjectivité qui se trouve engagée dans l'activité productive. Espérer se réaliser dans le travail, c'est faire de celui-ci l'espace où l'on met sans cesse à l'épreuve son estime de soi, où l'on cherche à faire reconnaître sa valeur individuelle, et où l'on investit par conséquent tous ses talents et son énergie. De plus, quand les barrières spatiales et temporelles qui séparaient jadis le travail de son extérieur sont estompées, le travail ne s'arrête jamais tout à fait : il englobe l'ensemble de l'existence et vient « miter » la vie familiale et les loisirs.

Le projet de Google est une représentation hyperbolique de ces évolutions. Le « lieu de travail » y devient l'espace d'un large spectre d'activités (du cours de yoga au jardinage écologique, de la dégustation d'un macchiato en terrasse aux calories brûlées sur un vélo) censées permettre à l'individu contemporain de se réaliser tout en ne délaissant jamais vraiment son entreprise. En d'autres termes, le projet de campus fait apparaître dans l'espace l'indistinction croissante entre différentes temporalités. À la fin des années 1960, Theodor Adorno pouvait encore écrire que même si le temps de nontravail n'avait au fond pour fonction que de reproduire la force de travail, la morale bourgeoise persistait à séparer avec un zèle puritain le travail du temps libre, celui-là 
ne devant en rien faire penser à celui-ci (Adorno 2003). Le capitalisme numérique, dont Google est l'incarnation, tend au contraire à abolir les frontières entre les différentes sphères de l'existence. Google semble ainsi donner raison aux théoriciens du " travail immatériel », qui remarquaient dès le début des années 1990 que le travail devenait une activité cognitive, créative et relationnelle, au sein de laquelle on ne pouvait plus guère " distinguer le temps de travail du temps de reproduction ou du temps libre » (Negri et Lazzarato 1991).

Le travail selon Google est donc bien cette activité biopolitique, qui s'étend sur tous les temps sociaux, implique l'ensemble du sujet, et produit aussi bien de la valeur économique que des formes de vie et des relations entre individus. Le projet architectural de Bjarke Ingels Group et T. Heatherwick en est l'incarnation parfaite. Il vise à recréer au sein d'un espace unifié et cohérent une société miniature, capable de répondre à l'ensemble des aspirations de l'individu, et donc de pousser celui-ci à ne jamais quitter son « lieu de travail » car ce dernier est devenu son lieu de vie.

\section{Un extérieur occulté}

17 L'utopie de Google a beau être totalisante, l'image ne montre pas tout. Cet extérieur invisible, voire " invisibilisé », est loin d'être insignifiant. Pour le comprendre, il faut rappeler que le nouveau complexe de Google a été pensé en priorité pour ses travailleurs les plus qualifiés (ingénieurs, développeurs, scientifiques), chargés de mettre au point les produits et services du futur. Si ces travailleurs sont bien entendu au cœur de la réussite de Google, l'entreprise de Mountain View ne prospère pas uniquement grâce à eux mais aussi grâce à d'autres formes de travail. Quelles sontelles?

Il y a tout d'abord le travail industriel, indispensable pour fabriquer tous les objets (ordinateurs, smartphones, objets connectés) sans lesquels l'économie " immatérielle » de Google n'existerait pas. Ce travail industriel n'a pas disparu, mais il est devenu relativement invisible pour les consommateurs européens et américains, dans la mesure où il a été délocalisé, essentiellement dans le sud-est asiatique. Il suppose luimême un travail encore plus fondamental et tout aussi invisible, si ce n'est davantage : celui d'extraire les minerais et métaux indispensables à la fabrication des ordinateurs et autres smartphones. Retrouver la trace de ce travail suppose de se rendre bien loin de Mountain View, essentiellement en Afrique où se trouve une part importante de ces ressources (Fuchs et Sandoval 2014).

Une dernière forme de travail invisible sur l'image est celui de chacun d'entre-nous, en tant qu'utilisateurs des services de Google. Il s'agit du fait que nous ne cessons de fournir à cette entreprise des données, personnelles ou non, qui lui permettent d'améliorer ses algorithmes et ses programmes d'intelligence artificielle, ainsi que d'engranger de gigantesques revenus tirés du marché de la publicité ciblée. Cette activité là n'est pas un travail au sens classique, puisque nous n'avons pas l'impression de travailler lorsque nous l'accomplissons et qu'elle se fait d'une certaine manière en dehors du champ de notre volonté et de notre conscience. Mais quel que soit le nom qu'on lui donne et la fonction qu'on lui assigne dans le processus de création de valeur, elle est assurément essentielle à la prospérité de la firme de Mountain View. 
La prise en compte de ces extérieurs fait finalement apparaître une polarisation du travail dans l'économie numérique, qui se joue au moins à deux niveaux. D'une part, la nouvelle division mondiale du travail a concentré les activité de création, de design et de conception dans quelques centres urbains (exemplairement, à San Francisco et dans la Silicon Valley) et les activités de fabrication industrielle dans les pays à faible coût de main d'œuvre. D'autre part, au sein des pays du Nord, l'économie numérique tend à creuser l'écart entre une minorité qui dispose d'emplois " créatifs » recherchés et bien payés (les ingénieurs de Google) et le reste de la population qui, en utilisant massivement les services en ligne, contribue aussi à les produire et à les enrichir, mais sans être rémunérée pour ce faire et sans véritable contrôle de l'usage qui est fait de toutes ces micro-contributions productives.

\section{Conclusion}

Plus qu'une utopie, le projet de campus Google représente finalement une " fantasmagorie ", au sens où Marc Berdet a développé ce concept à partir des écrits de Walter Benjamin. Il s'agit en effet d'un " lieu clos saturé d'imaginaire », cherchant à mobiliser les désirs par l'architecture (Berdet 2013, 8). À l'instar d'autres fantasmagories contemporaines (Disneyland, les shopping malls), c'est une hybridation de l'ancien et du moderne (la tradition américaine des campus universitaires revisitée à l'aune des prouesses de la Silicon Valley), qui expurge le présent de ses contradictions et occulte la réalité des conditions économiques. Un projet comme celui de Google participe donc bien du monde imaginaire du capitalisme, c'est-à-dire d'un ensemble de dispositifs destinés à susciter l'adhésion des élites tout en faisant rêver les masses.

\section{BIBLIOGRAPHIE}

Adorno, Theodor. 2003. « Temps libre ». In Modèles critiques, 205-217. Paris : Payot.

Barbrook, Richard \& Cameron, Andy. 1996. « The Californian Ideology ». Science as Culture, 6 (1), janvier $1996: 44-72$.

Berdet, Marc. 2013. Fantasmagories du capital. Paris : La Découverte, Zones.

Cingolani, Patrick. 2014. Révolutions précaires : essai sur l'avenir de l'émancipation. Paris :

La Découverte.

Dujarier, Marie-Anne. 2014. Le travail du consommateur. Paris : La Découverte.

Coase, Ronald H. 1937. « The Nature of the Firm ». Economica, New Series, vol. 4, n 16, novembre : 386-405.

Fuchs, Christian \& Sandoval, Marisol. 2014. « Digital Workers of the World Unite ! A Framework for Critically Theorising and Analysing Digital Labour ». tripleC, 12(2) : 486-563. http:// www.triple-c.at 
Jameson, Fredric. 2007. « L'utopie comme méthode ». ContreTemps, $\mathrm{n}^{\circ}$ 20, « Marx hors limites : une pensée devenue monde » :61-70.

Himanen, Pekka. 2001. L'éthique hacker. Paris : Exils.

Martin, Jérôme. 2013. « La vie privée ?'Peut-être une anomalie' selon un responsable de Google ». 22 novembre 2013. http://siliconvalley.blog.lemonde.fr/2013/11/22/la-vie-privee-peutetre-une-anomalie-selon-un-responsable-de-google/

Maurel, Lionel. 2018. « L'anti-protection sociale de Facebook et l'avènement du 'providentialisme de plateforme' ». S.I.Lex, 6 mai 2018. https://scinfolex.com/2018/05/06/lanti-protection-socialede-facebook-et-lavenement-du-providentialisme-de-plateforme/

Morozov, Evgeny. 2013. To Save Everything, Click Here. New York : Public Affairs.

Naddaff-Hafrey, Benjamin. 2016. « Work Imitates Life ». Aeon, 22 janvier 2016. https://aeon.co/ essays/is-the-utopian-workplace-just-a-ploy-to-keep-us-all-at-work

Negri, Antonio \& Lazzarato, Maurizio. 1991. « Travail immatériel et subjectivité ». Futur antérieur, $n^{\circ} 6$, mars : 86-89.

Oremus, Will. 2015. " Why Google's Hometown Said “No” to a Massive New Googleplex ». Slate, 7 mai 2015. http://www.slate.com/technology/2018/05/the-la-times-isnt-letting-europeans-readits-site-thanks-to-the-eus-new-internet-rules.html

Sadin, Éric. 2016. La Silicolonisation du monde. L'irrésistible expansion du libéralisme numérique. Paris : L'Échappée.

Simonet, Maud. 2010. Le travail bénévole. Engagement citoyen ou travail gratuit? Paris : La Dispute, coll. « Travail et salariat ».

Stone, Brad. 2015. « Big and Weird : The Architectural Genius of Bjarke Ingels and Thomas Heatherwick ». Bloomberg.com, 7 mai 2015. http://www.bloomberg.com/news/features/ 2015-05-07/google-s-new-campus-architects-ingels-heatherwick-s-moon-shot

Strickland, Jonathan. 2008. « How the Googleplex Works ». 8 avril 2008.

www.howstuffworks.com/googleplex.htm

Smyrnaios, Nikos. 2017. Les GAFAM contre l'Internet. Paris : INA Éditions.

Walzer, Michael. 2013. Sphères de justice. Paris : Seuil.

\section{RÉSUMÉS}

Cet article propose une réflexion sur la manière dont une entreprise emblématique de l'économie numérique, Google, conçoit l'avenir du travail. Cette réflexion est fondée sur l'analyse d'une image réalisée par le cabinet d'architectes et le studio de design en charge de concevoir le futur campus de l'entreprise. Au-delà des parti-pris singuliers des architectes, cet image nous semble révéler quelque chose de plus général : l'imaginaire que les géants de la Silicon Valley associent, ou veulent nous voir associer, au travail. Nous montrons ainsi comment Google souhaite créer autour de ses activités une véritable société miniature, transparente, où les frontières entre le travail et la vie s'estompent. Nous insistons également sur la manière dont cette fantasmagorie occulte un certain nombre d'autres réalités productives.

This article reflects on the way that a company at the forefront of the digital economy, Google, considers the future of work. It is based on the analysis of an image made by the group of 
architects and designers in charge of the company's future campus. I believe this image not only shows the architects' own choices and singularity, but also reveals something more general: the imaginary that Silicon Valley behemoths associate, or want us to associate, to work. The article shows how Google aims to create a transparent microsociety around its activities, where the boundaries between life and work are blurred. I also insist on the way this phantasmagoria casts a shadow over a great number of other productive activities.

El presente artículo propone una reflexión sobre la manera en que la emblemática empresa de la economía digital, Google, concibe el futuro del trabajo. Esta reflexión se funda en el análisis de una imagen realizado por la firma de arquitectos y el grupo de diseñadores a cargo de concebir el futuro campus de la empresa. Más allá de las ideas singulares de los arquitectos, esta imagen parece revelarnos un trasfondo más general : se trata del imaginario que los gigantes de Silicon Valley asocian, o quieren hacernos asociar, con el trabajo. Mostraremos aquí cómo Google busca crear en torno a sus actividades una verdadera sociedad en miniatura, transparente, donde las fronteras entre el trabajo y la vida se difuminan. Insistiremos igualmente en la manera en que esta fantasmagoría oculta un número importante de otras realidades productivas.

\section{INDEX}

Mots-clés : Avenir du travail, Google, Googleplex, transparence, imaginaire

Palabras claves : Futuro del trabajo, Google, Googleplex, transparencia, imaginario

Keywords : Futur of work, Google, Googleplex, Transparence, Imaginary

\section{AUTEUR}

\section{SÉBASTIEN BROCA}

Sociologue, Maître de conférences en Sciences de l'information et de la communication à l'Université de Paris 8. Il travaille sur les communs numériques et le digital labour. Il a publié Utopie du logiciel libre (Le passager clandestin, 2013).

Sociologist. He is currently Associate Professor in the Media and Communication Department at Université Paris 8 Vincennes Saint-Denis. He works on the digital commons and on digital labour. He has published Utopie du logiciel libre (Le passager clandestin, 2013) 